\title{
Amid COVID-19 pandemic - an innovative use of local resources to substitute the need for specialized operation theatre tables for orthopaedic and trauma surgery
}

\author{
Hemant Bansal $^{1}$ (D) Aashraya Karpe $^{2}$ (D) Samarth Mittal $^{1}$ (D) Vivek Trikha $^{1}$ (D)
}

Received: 5 June 2020 / Accepted: 26 July 2020 / Published online: 3 August 2020

(C) SICOT aisbl 2020

\begin{abstract}
Pelvic-acetabulum and spine surgery are two important constituents of complex trauma surgery and require a gamut of resources for fracture management. In the pandemic crisis of COVID-19 where existing health care resources are scarce and a major section of health care infrastructure has been assigned to fight against it, the compromised and less equipped health care facility is left to tackle the ongoing presentation of complex trauma surgery. One major challenge faced to manage these complex procedures is the availability of specialized radiolucent operation tables as even the apex level health care setups generally have one or two such kinds of table. Therefore, in this state of pandemic crisis, innovations utilizing the available local resources need to be developed to avoid deferring these complex operations.
\end{abstract}

Keywords Operation table $\cdot$ COVID- $19 \cdot$ Coronavirus $\cdot$ Orthopaedics $\cdot$ Spine $\cdot$ Pelvic-acetabulum

\section{Introduction}

Severe acute respiratory syndrome coronavirus 2 (SARS-CoV2), a novel coronavirus, has recently emerged as a lethal pathogen for humans worldwide and the cause for 2019 novel coronavirus disease (COVID-19) throughout the globe [1, 2]. The World Health Organization (WHO) has declared it a pandemic disease considering worldwide affection and with a steep rise in the positive number of cases. So far globally, more than two million people are affected and more than a hundred thou-

Vivek Trikha

vivektrikha@rediffmail.com

Hemant Bansal

hemant.21bansal@gmail.com

Aashraya Karpe

aashrayak@gmail.com

Samarth Mittal

SAMARTHMITTAL@gmail.com

1 Department of Orthopaedics, JPNApex Trauma Centre, AIIMS, New Delhi, India

2 Shroff Eye Hospital, New Delhi, India sand are dead. All nations are fighting at their own level to combat this deadly COVID-19 and the main segment which has been challenged and fighting for is the health care system. The major crisis encountered during this pandemic is to distribute the existing health care facility into various sections where priority is given to services reserved for the COVID-19 task force.

In the majority of nations, the health care infrastructure is not evolved enough to deal with COVID-19 and existing health issues simultaneously. Therefore, under such circumstances, it is the "need of the hour" to utilize the available resources for effective and efficient management of health-related issues.

There has been a drastic decline in the number of road traffic accidents (RTAs) and the incidence of trauma cases due to lockdowns across countries. However, cases due to falling from a height, trivial trauma at home, and regional assault have prevailed and highvelocity RTAs have increased due to relatively lower traffic rates. Therefore, the management of such cases amid the COVID-19 pandemic is challenging.

To combat COVID-19 and existing health-related issues simultaneously, health care facilities have been divided into two separate halves wherein one part looks after COVID positive cases while others look at COVID negative cases. Both these groups of patients can suffer from traumatic injuries and due to lack of double resources, it is difficult to manage both kinds of cases with the existing specialized equipment. 
Fig. 1 Picture depicting the standard OT table with broad radio-opaque column hindrance in visualizing the pelvicacetabulum and lower $2 / 3$ rd spine

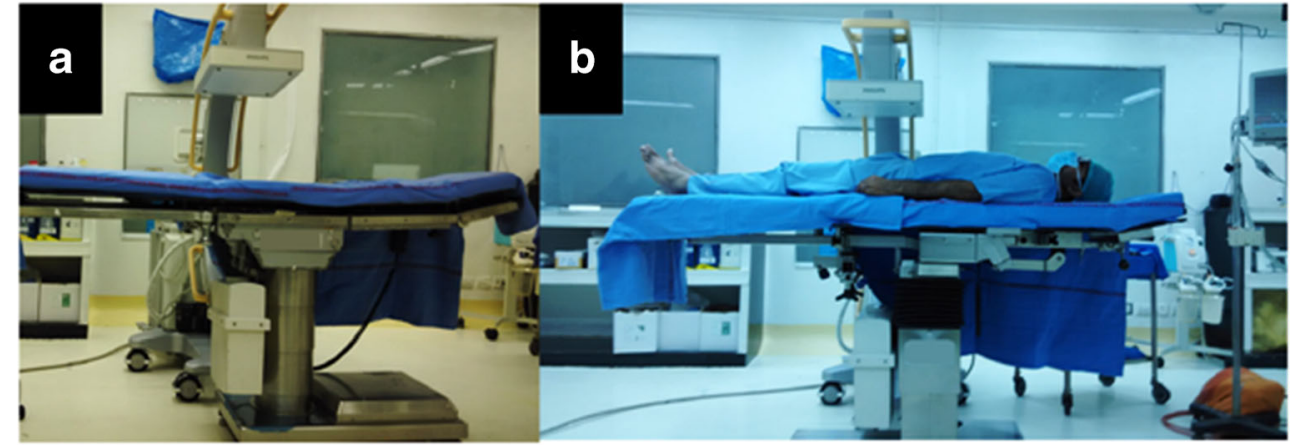

Spine and pelvic-acetabular trauma surgery need specialized radiolucent operation theatre (OT) tables which allow unhindered use of image intensifier during the internal fixation of fractured bone with various implants. The majority of trauma centres may have only one or two specialized operation theatre tables to deal with these complex trauma operations. When resources are divided physically into two halves for the distancing of patients, OT complexes have also been divided into many health setups in two halves where one half is meant for surgery for COVID positive patients while the other half is meant for operating COVID negative patients. Division of operating tables would be done in between these areas and one of these areas may or may not have such specialized tables. Also, during this pandemic crisis, when such specialized OT tables get malfunctioned, it may not be possible to repair them in the current context given the international cargo and manpower travel restrictions as well as parts availability with the closing of factories producing them.

Therefore, we propose an innovative method of utilizing the available local resources in OT premises, applicable in all kinds of OT setups to substitute the need for such specialized OT tables, if required.

\section{Operating tables}

Routinely, the standard OT table used in most of the trauma centres and orthopaedic centers has a broad radio-opaque column underneath to support the top of the table, which occupies most of the head end of the top of table hindering the visualization of pelvic-acetabulum and lower $2 / 3$ of the spine under the image intensifier (Fig. 1), thus, compromising

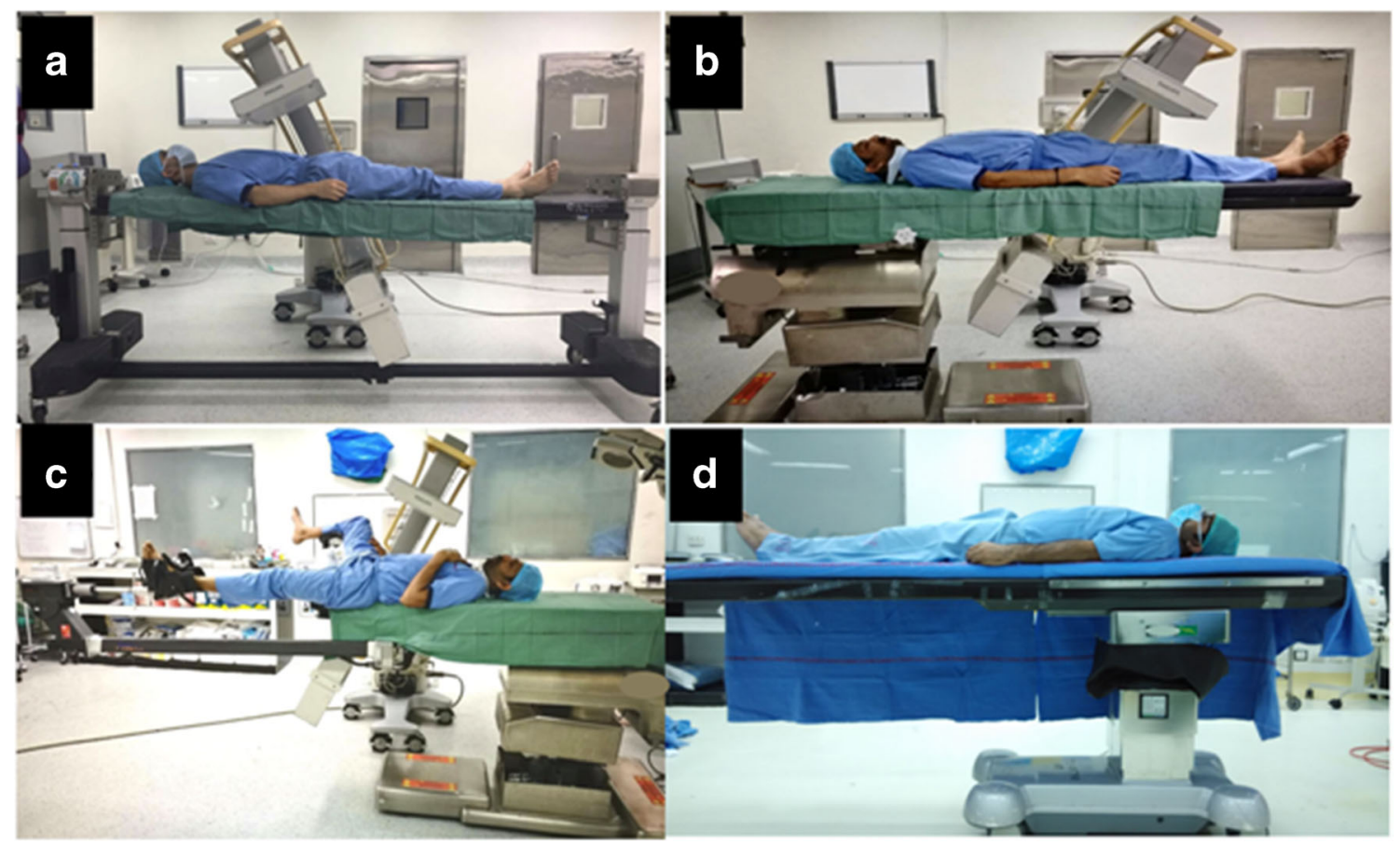

Fig. 2 Specialized OT tables: a modular radiolucent table; b profracture radiolucent imaging table; $\mathbf{c}$ pelvic reconstruction orthopaedic table; $\mathbf{d}$ spine radiolucent table with sliding option 


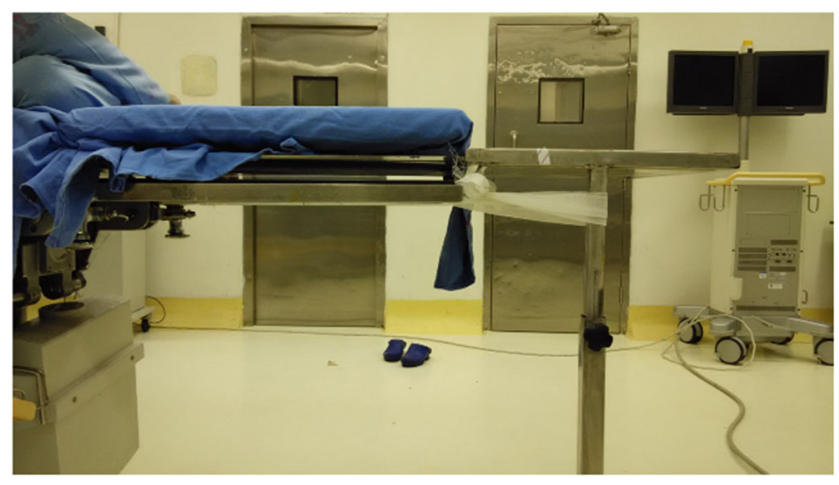

Fig. 3 Picture depicting use of Mayo stand for extension of foot end of standard OT table

accurate fracture reduction and fixation. To avoid such hindrance in visualizing the above-concerned areas, few specialized designs are available with customized completely radiolucent top of the table to facilitate easy image intensifier mobilization intra-operatively (Fig. 2). These specialized tables due to their sophisticated, unique design carry a huge economic burden in the OT setups, especially in most developing countries, and are not widely available throughout all centers.

\section{New innovative method}

The major restriction with the use of the standard OT table is the broad radio-opaque table column beneath the upper end of the top of the table hindering with visualization under image intensifier. To avoid this hindrance, we used an extension at the foot end of the top of the table to facilitate the downward positioning of the patient torso evading the radio-opaque table column.

Mayo stands are readily available in the operating rooms, so we decided to make use of its large and strong platform for foot end extension of the table. Mayo stand was tied with bandages to the foot end of the table, after adjusting its height to the level of the OT table (Fig. 3). Silicon pads available in OT, routinely used for cushioning bony prominence, were used to cover the metallic surface of Mayo stand and to cushion the foot end. Thick folded sheets available in OT were also used for further cushioning the foot end and leveling the extension with the OT table. The height of the Mayo stand can be adjusted intra-operatively with the OT table using a height adjustment knob in the stand.

This facilitated positioning of the patient in such a way that the patient's headrests over the radio-opaque table column and torso over the lower radiolucent half of the table. The whole of the pelvic-acetabulum and spine could, thus, be easily visualized under image intensifier in both supine and prone position (Fig. 4).

In extreme scenarios when even Mayo stand is not available, then one can execute the surgery of the pelvic-acetabulum or spine by making use of two standard OT tables in such a way that they are positioned with the foot end of tables touching each other at the same level (Fig. 5). This positioning of two OT tables though, encasing large floor area, provides a complete radiolucent field for image intensifier to mobilize under and effective visualization of fracture site in different views.
Fig. 4 Picture depicting positioning of patient centered over radiolucent top of standard OT table with ease in image intensifier positioning. a Inlet pelvic view; b iliac oblique view of right hemi pelvis

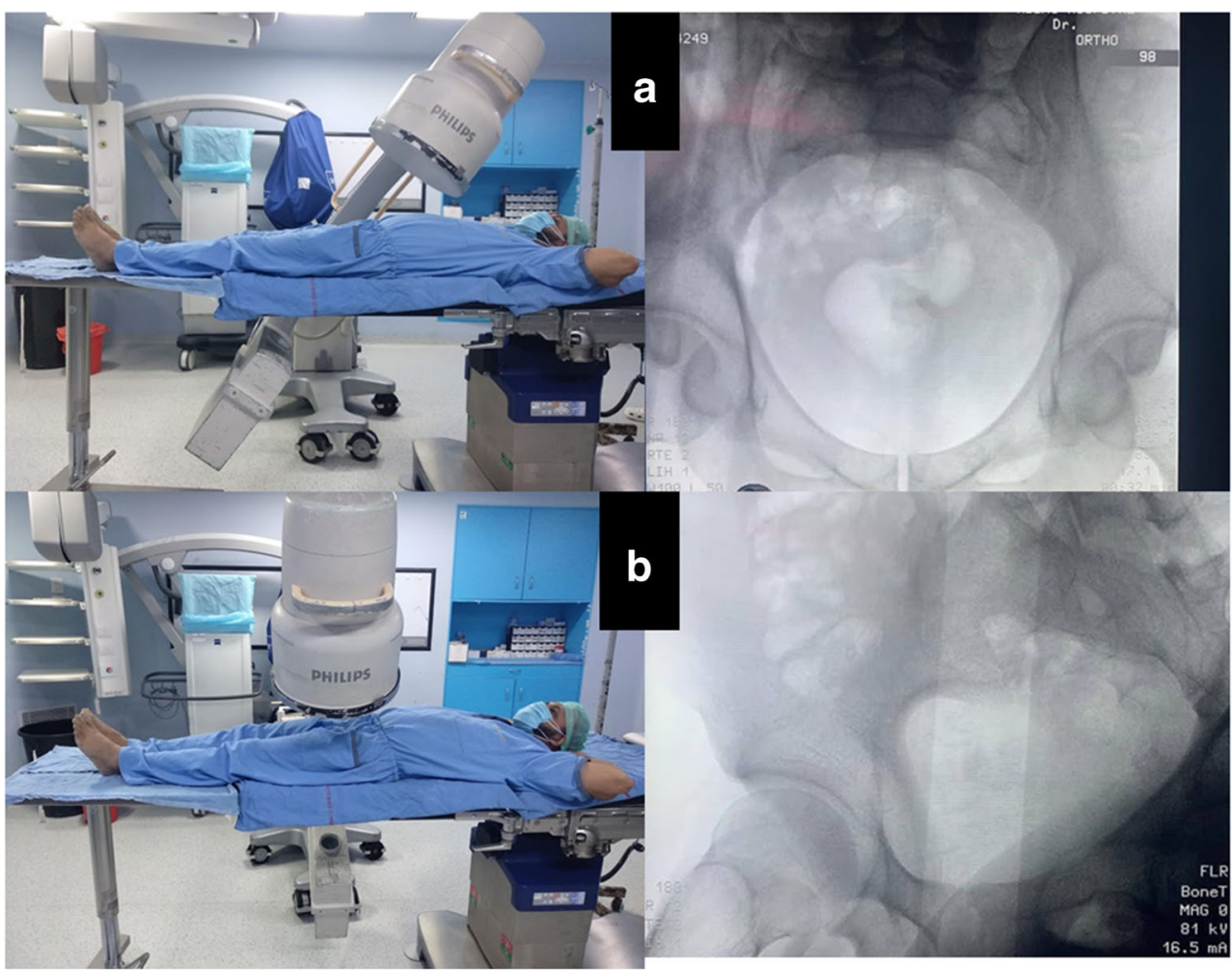




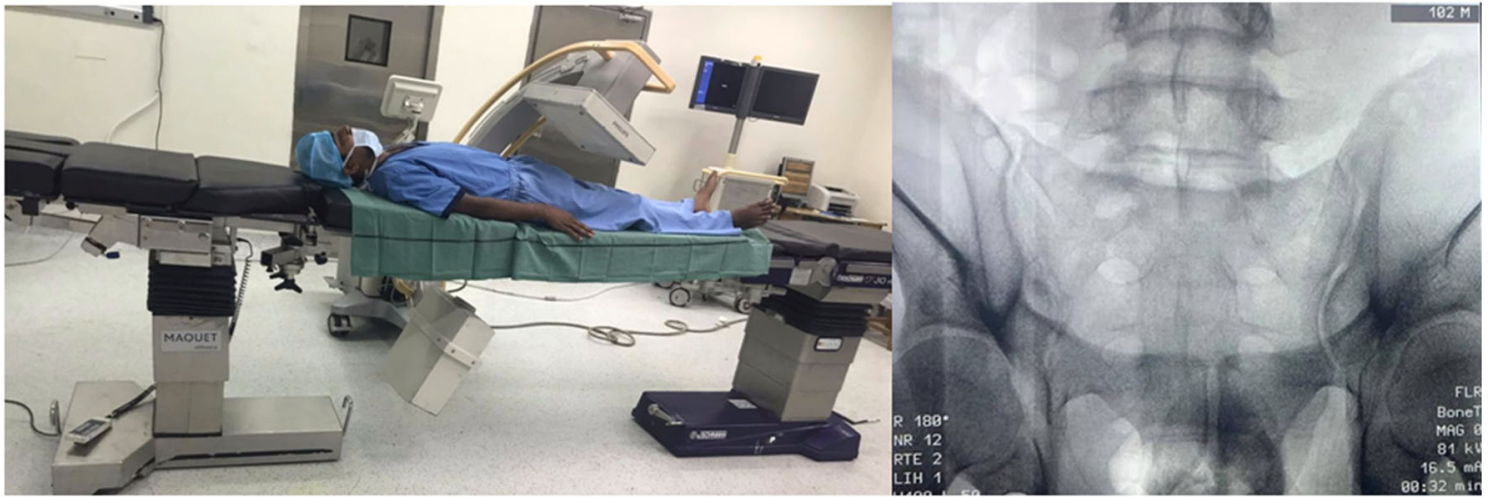

Fig. 5 Picture depicting use of two standard OT tables with foot ends facing each other, allowing central complete radiolucent surface for image intensifier positioning; outlet pelvic view is shown

\section{Discussion}

Pelvic-acetabular and spine surgery constitute an important aspect of orthopaedic trauma surgery. These operations are complex with the requirement of several additional resources. Under the crisis of COVID-19 pandemic, managing pelvicacetabular and spine injury is an immense challenge, especially in resource-limited settings.

One of the important aspects of managing such surgery is the need to accurately identify bony landmarks, fracture fragment reduction, and internal fixation. Visualization of fractures under image intensifier is a must in all modes of fixation and each procedure. Pelvic anatomy is complex and has very close proximity to intra-pelvic vital structures [3], which is also true for the spinal canal in spine surgery. Image intensifier guides in the placement of adequate sized pedicle screws in spine surgery and proper assessment of screw placement in narrow corridors of the pelvic bone. It also aids in confirming the position of multiple curved plates used for internal fixation of acetabular and pelvic fractures [4].

To use imaging intra-operatively, the operating table needs to be radiolucent. Various modifications need to be done in the operating table as well as in the image intensifier to visualize the whole of the pelvic-acetabulum and affected spine segment during surgery. In the standard OT tables, it becomes too challenging to get different radiographic views, especially pelvic inlet and outlet views due to hindrance offered by broad radio-opaque table column in image intensifier positioning and mobilization. In spine surgery, true anteroposterior, lateral, and oblique views must assess the accurate placement of pedicle screws which can only be facilitated by the completely radiolucent OT table and freely mobile image intensifier.

Spine injuries may present from a back sprain to spine fracture-dislocations with a significant injury to the spinal cord, leading to paraplegia or quadriplegia depending upon the level of the spinal cord affected. These injuries are real emergencies and surgery cannot be deferred due to unavailability or compromised health care setup [5]. Several litigations have been reported because of the wrong level of spinal surgery in the past [6]. In minimally invasive and percutaneous techniques, clinical anatomical landmarks cannot be used for the identification of the spinal level [7]. Thus, intra-operative use of image intensifier is of utmost importance in such a case.

In the modern era, various types of specialized designs of OT tables are commercially available that can provide various benefits including complete radiolucency enabling access to the whole of the area under image intensifier. However, these specialized tables are not economical and add burden to already compromised health care expenditure amid the pandemic crisis of COVID-19. Most of these specialized OT tables are imported from other countries and require quality checks and assessment of sophisticated machinery involved at regular intervals by the service personnel from the country of manufacturing, which is practically not possible currently amid worldwide lockdowns and international travel restrictions.

Therefore, we felt the need for a modification in the conventional standard OT table to avoid deferring the surgeries and patient care to such types of complex fracture patterns.

\section{Conclusion}

A pandemic crisis like COVID-19 is not anticipated and one has to be ready to utilize the alternate existing resources to provide the best possible health care to the patients. With such simple innovation, the need for specialized OT tables can be substituted with standard tables with readily available resources.

Acknowledgments We would like to acknowledge the help provided by Dr. Vivek Tiwari, Dr. Pankaj Sharma, and Dr. Rahul Yadav in helping with the preparation of manuscript as well as the images for the manuscript.

Authors' contributions Conceptualization: Vivek Trikha. Design: Hemant Bansal, Aashraya Karpe. Formal analysis and interpretation: 
Samarth Mittal, Hemant Bansal, Aashraya Karpe. Writing —original draft preparation: Hemant Bansal, Aashraya Karpe. Writing - review and editing: Hemant Bansal, Vivek Trikha, Samarth Mittal. Supervision: Vivek Trikha, Samarth Mittal.

\section{Compliance with ethical standards}

Conflict of interest The authors declare that there is no conflict of interest.

Ethical approval Ethical approval not required as per our Institute Review Board. All procedures performed in this study involving human participants were in accordance with the ethical standard of the institutional and/ international research committee and with the 1964 Helsinki declaration and its later amendments or comparable ethical standards.

\section{References}

1. Rothan HA, Byrareddy SN (2020) The epidemiology and pathogenesis of coronavirus disease (COVID-19) outbreak. J Autoimmun 26: $-102433$
2. Jain VK, Vaishya R (2020) COVID-19 and orthopaedic surgeons: the Indian scenario. Trop Dr 21:0049475520921616

3. Yi C, Burns S, Hak DJ (2014) Intraoperative fluoroscopic evaluation of screw placement during pelvic and acetabular surgery. J Orthop Trauma (1):28, 48-56

4. Giannoudis PV, Tzioupis CC, Pape HC, Roberts CS (2007) Percutaneous fixation of the pelvic ring: an update. J Bone Joint Surg Br Vol 89(2):145-154

5. Cengiz ȘL, Kalkan E, Bayir A, Ilik K, Basefer A (2008) Timing of thoracolumber spine stabilization in trauma patients; impact on neurological outcome and clinical course. A real prospective (rct) randomized controlled study. Arch Orthop Trauma Surg 128(9):959966

6. Hsiang J (2011) Wrong-level surgery: a unique problem in spine surgery. Surg Neurol Int, 2

7. Reiley M, inventor, Reiley Mark A, assignee (2005). Percutaneous spine distraction implant systems and methods. United States patent application US 11/041,570. Jul 28

Publisher's note Springer Nature remains neutral with regard to jurisdictional claims in published maps and institutional affiliations. 